\title{
New Opportunities for Diagnosis and Prognosis of Stroke: The Benefits of Across Border Approaches
}

\author{
Denis Vivien ${ }^{1,2}$ \\ ${ }^{1}$ Normandie Univ, UNICAEN, INSERM UMR-S U1237, Physiopathology \\ and Imaging of Neurological Disorders (PhIND), Institute Blood and \\ Brain@ Caen-Normandie (BB@C), GIP Cyceron, Caen, France \\ ${ }^{2} \mathrm{CHU}$ Caen, Department of Clinical Research, CHU Caen Côte de \\ Nacre, Caen, France
}

\begin{abstract}
Address for correspondence Denis Vivien, PhD, Normandie Univ, UNICAEN, INSERM UMR-S U1237, Physiopathology and Imaging of Neurological Disorders (PhIND), Institute Blood and Brain @ CaenNormandie (BB@C), GIP Cyceron, 14000 Caen, France (e-mail: vivien@cyceron.fr).
\end{abstract}

Hämostaseologie 2021;41:22-24.

\author{
Abstract \\ Keywords \\ - stroke/prevention \\ - imaging \\ - animal models \\ - proteases
}

Ischemic stroke is a leading cause of disability, with its treatment not yet optimal. It is thus mandatory to make preclinical research on this topic more efficient. This review summarizes current development of research aimed to improve diagnosis and prognosis of ischemic stroke. For more details, see our recent review published in Lancet Neurology.

\section{Introduction}

Ischemic stroke remains one of the leading causes of disability and the cause of death in western countries. Stroke incidence is expected to increase in the coming years, with an estimated $34 \%$ increase in strokes by 2035 in the European Union. Among the various subtypes of stroke, acute ischemic stroke (AIS) consecutive to clots obstructing large intracranial arteries currently represent the majority of strokes responsible for death and disability. For this subtype of stroke (i.e., AIS following a clot obstruction), time is critical. For every 30 minutes spent to reopen the artery, mortality increases by $20 \%$. Rapid recanalization (i.e., reopening of the arteries) is therefore compulsory. The clots responsible for occlusion of the large intracranial arteries are often resistant to intravenous thrombolysis ( $\sim 70$ to $80 \%$ of cases), which is the current gold standard for medical therapy. In addition to the lack of efficacy, thrombolysis is associated with a risk of intracranial hemorrhage responsible for increased mortality in the first week after the stroke. Mechanical thrombectomy (MT; i.e., the removal of the thrombus by the endovascular route) is part, in addition to intravenous thrombolysis, of the recommendation for care. Despite this combined therapy, more than $50 \%$ of patients after MT will remain disabled. ${ }^{1}$

Tissue-Type Plasminogen Activator and New Fibrinolytics Although recent technical advances in thrombectomy have revolutionized acute stroke treatment, prevalence of disability and death related to stroke remain high. Today, plasminogen activators-which can promote fibrinolysis by converting plasminogen into active plasmin and facilitate clot breakdown-are still commonly used at the acute phase of ischemic stroke. Hence, plasminogen activators, including tissue-type plasminogen activator (tPA), have become a crucial area for clinical investigations for their ability to recanalize occluded arteries in ischemic stroke and to accelerate hematoma clearance in hemorrhagic stroke. However, inconsistent results, insufficient evidence of efficacy, or reports of side effects in trial settings might reduce the use of plasminogen activators in clinical practice. This leads to an active research to improve its efficacy or to the development of alternative strategies to promote thrombolysis.

Additionally, the mechanism of action for plasminogen activators extends beyond the vessel lumen and involves plasminogen-independent processes, which support the idea that plasminogen activators have also non-fibrinolytic roles. For example, tPA is known to activate a set of receptors, such as the low-density lipoprotein receptor protein, the $\mathrm{N}$-methyl-D-aspartate (NMDA) receptors, epidermal growth factor receptor, or annexin-II, and to promote cleavage of either pro-BDNF (brain-derived neurotrophic factor) or of pro-PDGF-CC (platelet-derived growth factor-CC) into their respective active forms. By these mechanisms, tPA was involved in the regulation of the neuronal migration, received

August 21, 2020

accepted after revision

December 17, 2020 (c) 2021. Thieme. All rights reserved. All DOI https://doi.org/ rights reserved.

Georg Thieme Verlag KG,

Rüdigerstraße 14,

70469 Stuttgart, Germany 
synaptic plasticity, neuronal survival, control of the integrity of the blood-brain barrier, learning and memory processes, or anxiety behavior. ${ }^{2}$ Understanding the complex mechanisms of action of tPA in the brain, including those occurring at stroke onset and later, could guide future directions for diagnosis (brain imaging, biomarkers) and therapeutic interventions in patients with ischemic and hemorrhagic stroke.

Optimization of Experimental Ischemic Stroke Models We are convinced that the failure of translation from preclinical investigations to clinic is due at least in part to the unrelevance of our experimental models. Among the main differences between what happens in clinic and what we are doing in our laboratory, is the use of anesthesia for experimental models, with anesthetics targeting pathways highly involved in the pathophysiology of stroke, such as NMDA receptors and GABA receptors. To bypass this gap, we have developed a thromboembolic stroke model in awake mice, compatible with awake brain imaging (magnetic resonance imaging [MRI], functional ultrasound (fUS)/ultrasound localization microscopy (ULM); see section "New Imaging Modalities"). Interestingly, correlations between cerebral blood flows and lesion volumes or functional outcomes were observed only when the stroke occurs awake. This is particularly interesting since correlation between cerebral blood flow and final outcome is a pre-requirement to make preclinical studies relevant with the clinic. Comorbidities, including diabetes, high blood pressure, and aging, are also considered. Accordingly, in the context of increasing recognition of the importance of multicentric preclinical trials, there is a need for a widely applicable and clinically relevant experimental model, mimicking as much as possible the pathophysiology of human stroke. Another important point to explain the failure of translational research in the field of stroke is a lack of knowledge of the pathophysiology of stroke, comparing with the clinical settings, reinforced by the multitude of animal models available. In this context, we are studying the differences in pathophysiology between experimental stroke models and human stroke, thanks to our expertise going from in vitro experiments on neurons to in vivo brain imaging in humans and fruitful discussions between basic researchers and clinicians.

\section{New Treatments for Ischemic Stroke}

The main limitations of tPA administration in AIS are its low rate of recanalization $(<30 \%)$, the risk of symptomatic parenchymal hemorrhage $(\sim 6 \%)$, and the risk of symptomatic brain edema. Regarding the low rate of recanalization, we are exploring strategies to increase the fibrinolytic activity of tPA (mutant of tPA, coadministration with a clinically available plasmin activity boosting molecule defibrotide, etc.) and to target other constituents of the thrombus (von Willebrand factor [VWF] targeting treatment such as N-Acetylcystein (NAC) and its derivatives-glutathione, carboxy-cysteine, antiplatelet, and anticoagulant agents). To reduce the proedema effect of tPA, which we and other demonstrated to be plasmin and bradykinin dependent, we are working on a combinatory strategy using tPA and antagonists of bradyki- nin receptor (B1 and B2), inhibitors of factor XII, and highmolecular-weight kininogen. ${ }^{3}$ Among the other strategies that we are investigating, we are particularly interested to test new neuroprotective drugs (e.g., Glunomab) ${ }^{4}$ and antiinflammatory drugs with a particular interest for perivascular macrophages. Our current data suggest that perivascular macrophages play a key role in the control of the routes lymphocytes use to invade the brain when it is injured. Once the efficacy of these treatments will be demonstrated in experimental models, we plan to perform multicentric preclinical trials to validate their efficacy before moving to clinic. Among our most advanced treatments is $\mathrm{N}$-acetylcysteine, ${ }^{5}$ which we will soon be tested in a randomized trial in AIS.

\section{Molecular Imaging of Adhesion Molecules}

These last years, we have contributed to the field of brain imaging by developing, characterizing, and sharing an innovative methodology for high-resolution MRI of adhesion molecules using Micron-sized particles of iron oxide (MPIOs), including VCAM-1, P-selectin, and MAdCAM-1. We demonstrated the applicability of this method to improve the diagnostic performance of MRI in several neurovascular, neurological, cardiovascular, and autoimmune diseases. $^{6-9}$ These methods of molecular MRI has now been widely adopted for preclinical research and warrant further development toward clinical application. Unfortunately, currently available MPIOs are not biodegradable and therefore not usable in humans. The next step, beyond further extending the applications and molecular targets of this technology, is to develop biocompatible MPIOs.

\section{Imaging of Thrombi}

In vivo molecular imaging of the thrombus is an important topic for precision medicine, which is able not only to detect the thrombi responsible for vessel occlusion but also to noninvasively collect data about their structure (fibrin and platelet content, VWF, and neutrophil extracellular trap detection). In the context of AIS, it could allow to adapt the thrombolytic strategy to each patient (What cocktail of thrombolytic agent should be used? What endovascular device?). To achieve this goal, our group is developing innovative tools, first based on an original phenomenon called passive retention of micro-sized particles in microthrombi (patent under deposit) allowing to detect microthrombi downstream of the main thrombus in the context of AIS; second using biocompatible MPIOs targeting either platelets, VWF, histone, and/or fibrin. Using the different experimental models of thrombosis available, imaging of thrombi displaying varying structures can be performed, allowing to validate each of these tools.

\section{New Imaging Modalities}

Although $1 \mathrm{H}-\mathrm{MRI}$ is very useful in research and in clinic, we are contributing it to the development of emerging and complementary imaging modalities, such as Ultrafast ultrasound in vivo microscopy imaging (fUS/ULM). Using fUS/ULM, we have provided data demonstrating that early fUS/ULM imaging ( $<2$ hours after stroke onset or treatments) 
could be useful to predict final outcomes following stroke (in rodents) including responses to treatments, usually observed only after 24 to 48 hours. ${ }^{10}$ We are now working to translate this methodology to the clinical setting.

\section{Conflict of Interest}

The authors declare that they have no conflict of interest.

\section{References}

1 Powers WJ, Rabinstein AA, Ackerson T, et al. Guidelines for the early management of patients with acute ischemic stroke: 2019 update to the 2018 guidelines for the early management of acute ischemic stroke: a guideline for healthcare professionals from the American Heart Association/American Stroke Association. Stroke 2019;50(12):e344-e418

2 Thiebaut AM, Gauberti M, Ali C, et al. The role of plasminogen activators in stroke treatment: fibrinolysis and beyond. Lancet Neurol 2018;17(12):1121-1132

3 Marcos-Contreras OA, Martinez de Lizarrondo S, Bardou I, et al. Hyperfibrinolysis increases blood-brain barrier permeability by a plasmin- and bradykinin-dependent mechanism. Blood 2016; 128(20):2423-2434
4 Lesept F, Chevilley A, Jezequel J, et al. Tissue-type plasminogen activator controls neuronal death by raising surface dynamics of extrasynaptic NMDA receptors. Cell Death Dis 2016;7(11):e2466

5 Martinez de Lizarrondo S, Gakuba C, Herbig BA, et al. Potent thrombolytic effect of $\mathrm{N}$-acetylcysteine on arterial thrombi. Circulation 2017;136(07):646-660

6 Gauberti M, Fournier AP, Docagne F, Vivien D, Martinez de Lizarrondo S. Molecular magnetic resonance imaging of endothelial activation in the central nervous system. Theranostics 2018;8(05):1195-1212

7 Quenault A, Martinez de Lizarrondo S, Etard O, et al. Molecular magnetic resonance imaging discloses endothelial activation after transient ischaemic attack. Brain 2017;140(01):146-157

8 Fournier AP, Quenault A, Martinez de Lizarrondo S, et al. Prediction of disease activity in models of multiple sclerosis by molecular magnetic resonance imaging of P-selectin. Proc Natl Acad Sci U S A 2017;114(23):6116-6121

9 Fournier AP, Martinez de Lizarrondo S, Rateau A, et al. Ultrasensitive molecular imaging of intestinal mucosal inflammation using leukocyte-mimicking particles targeted to MAdCAM-1 in mice. Sci Transl Med 2020;12(560):4047

10 Hingot V, Brodin C, Lebrun F, et al. Early ultrafast ultrasound imaging of cerebral perfusion correlates with ischemic stroke outcomes and responses to treatment in mice. Theranostics 2020; 10(17):7480-7491 